\title{
The contribution of Magnetic Resonance I maging in the differential diagnosis between leiomyoma typical, atypical and uterine sarcomas: Personal experience
}

\author{
Oscar Tamburrini ${ }^{1}$, Claudia Smiraglio ${ }^{1}$, Nicoletta Caputo ${ }^{1}$, Nadia Mastroianni ${ }^{1}$, Giuseppe \\ Guarascio $^{1}$, Di Cello Annalisa ${ }^{2}$, Michele Morelli ${ }^{2}$, Fulvio Zullol ${ }^{2}$ \\ 1. Department of Experimental and Clinical Medicine-Radiology, University of Catanzaro, Italy. 2. Department of \\ Obstetrics and Gynaecology, University of Catanzaro, Catanzaro, Italy
}

Correspondence: Claudia Smiraglio. Address: Department of Experimental and Clinical Medicine-Radiology, University of Catanzaro, Viale Europa, Località Germaneto, Catanzaro 88100, Italy. Email: claudia.smiraglio1@gmail.com

Received: April 5, 2016

DOI : $10.5430 /$ ijdi.v3n2p71
Accepted: June 13, 2016

Online Published: July 18, 2016

URL: http://dx.doi.org/10.5430/ijdi.v3n2p71

\section{Abstract}

Purpose: Evaluate the role of Magnetic Resonance Imaging (MRI) in the differential diagnosis between degenerated leiomyomas, cellular leiomyomas and uterine sarcomas.

Materials and methods: From February 2015 to August 2015, 42 patients were enrolled, aged between 18 and 75 years, suffering from uterine "mass” waiting for surgery and considered at risk and/or uncertain clinical and laboratory investigations and imaging. These patients were submitted to MRI exam of the pelvis that was performed by morphological study, DWI sequences and dynamic sequences post-contrastographic.

Results: The analysis of MRI images of the 42 patients made possible to do a diagnosis of uterine sarcomas in 8 patients with the aid of sequences weighted in diffusion and dynamic, proving a sensitivity of 100\% and a specificity of $88 \%$.

Conclusions: Based on our experience, we may consider that by morphological study with DWI sequences weighted in diffusion and dynamic post-contrastographic sequences, the MRI is a working aid in the differential diagnosis between degenerated leiomyomas and uterine sarcomas.

\section{Keywords}

Uterus, Uterine sarcomas, Degenerated leiomyoma, Cellular leiomyoma, Magnetic Resonance Imaging

\section{I ntroduction}

Leiomyomas are the most common benign uterine tumors and the most frequent benign gynecological tumors. They occur in more than $20 \%$ of women older than 30 years ${ }^{[1]}$. They are predominantly composed of smooth muscle cells separated by variable amount of fibrous connective tissue. Exuberant development of these formations, not supported by an adequate blood supply, implicates different types of degeneration: hyaline, myxoid, calcified, cystic and red. Leiomyomas are classified in submucosal, intramural and subserosal; these last ones can be pedunculate and in some cases simulate an ovarian malignancy. Although the leiomyomas are asymptomatic in most of cases, the presence of one or more submucosal fibroids can determine menometrorrhagia or be an obstacle to reproduction (greater rate of abortions); less 
frequently the presence of very large leiomyomas may be linked to pressure symptoms on the surrounding structures or to abdominal pain ${ }^{[2,3]}$.

A particular subtype of leiomyomas is represented by cellular leiomyomas which are composed of compact smooth muscle cells and by a poor or absent connective component ${ }^{[4]}$. The uterine sarcomas are rare tumors representing approximately $1 \%$ of female genital neoplasms and $3 \%-7 \%$ of all uterine cancers ${ }^{[5]}$. Histologically, they are divided into carcinosarcomas, endometrial stromal sarcomas, leiomyosarcomas (LMS), undifferentiated stromal sarcomas and mullerianadenosarcomas ${ }^{[6]}$. The complete excision of the tumor is the only effective treatment modality of uterine sarcomas ${ }^{[7]}$.

In April 2014, the Food and Drug Administration (FDA) in a safety communication, has discouraged the use of morcellator during routine laparoscopic myomectomy or hysterectomy. The warning follows the outcry provoked all around the world for some cases of secondary sarcoma localization occurred, due to the dissemination of cancer cells from uterine sarcomas mis-diagnosed and underwent to laparoscopic morcellation ${ }^{[8-11]}$.

Since we are still lacking a sufficiently sensitive and specific strategy that enable to differentiate preoperatively a myoma from a sarcoma, and there are no signs or pathognomonic symptoms of sarcoma, in a $0.2 \%-1 \%$ of the patients, the diagnosis of uterine sarcoma is set retrospectively on the basis of a definitive histologic diagnosis in patients undergoing a surgery for a clinical suspicion of myoma. In these cases that are not preoperatively diagnosed, the use of electronic morcellator could increase the stage of the disease, encouraging the intra-abdominal dissemination of neoplastic cells, and worsen the prognosis. Thus it is clear the need for an accurate preoperative differential diagnosis between myoma and sarcomas $^{[7]}$.

Although ultrasound represents the first instance investigation in the study for imaging of the female pelvis, it has some limitations in revealing large tumors, due to a limited field of view, and in tissue characterization; moreover there is a significant overlap between the sonographic appearance of degenerated leiomyomas and that of the sarcomas ${ }^{[12-18]}$.

Magnetic Resonance Imaging (MRI) is the most accurate imaging method for the detection and localization of leiomyomas. On T2 images typical leiomyomas appear as circumscribed hypointense masses compared to myometrium ${ }^{[2]}$, while sarcomas appear as large iso-hyperintense masses. The same appearance is shown by cellular leiomyomas and some types of degenerated leiomyomas, which then may mime sarcomas. Therefore, the differential diagnosis between myoma and sarcoma can be difficult if it is just based on conventional sequences without administration of contrast agent ${ }^{[19-21]}$.

Diffusion Weighted Imaging (DWI) in recent studies has shown promising results in the differential diagnosis between benign and malignant gynecological tumours ${ }^{[22-26]}$. Nevertheless, using DWI in the diagnosis of uterine sarcomas is still limited. Particularly significant are also the post-contrastographic sequences that showed how sarcomas present a quick and avid enhancement compared to benign lesions ${ }^{[27-28]}$. Recently, according to some authors, the use of post-contrastographic-sequences can provide a more accurate information rather than sequences weighted in diffusion and it is preferable to DWI ${ }^{[29]}$.

Upon these considerations, the aim of our study is to confirm the value of MRI and, especially, of DWI and dynamic post-contrastographic-sequences in the differential diagnosis between degenerate myomas, cellular myomas and sarcomas.

\section{Materials and methods}

This is a prospective study carried out with the collaboration of the Division of Gynecology and Obstetrics of the University “Magna Graecia” of Catanzaro. 


\subsection{Population}

From February 2015 to August 2015, 42 patients aged between 18 and 75 years have been enrolled. Clinical findings and symptoms are shown in Tables 1 and 2.

Table 1. Chief symptoms of the study patients

\begin{tabular}{llll}
\hline Chief symptoms & Leiomyomas (30 cases) & Sarcomas $^{[8]}$ & Adenomyosis and malformation of mullerian ducts $^{[4]}$ \\
\hline Hypermenorrhea & 20 & 5 & 5 \\
Anemia & 20 & 7 & 3 \\
Abnormal genital & 5 & 3 & 0 \\
bleeding & 14 & 2 & 3 \\
Dysmenorrhea & 10 & 3 & 2 \\
Prolonged menses & 10 & 4 & 2 \\
Sterility & & &
\end{tabular}

Table 2. Clincal findings of the study patients

\begin{tabular}{|c|c|c|c|}
\hline Variable & $\begin{array}{l}\text { Leiomyomas } \\
\text { (30 cases) }\end{array}$ & Sarcomas ${ }^{[8]}$ & $\begin{array}{l}\text { Adenomyosis and malformation } \\
\text { of mullerian ducts }{ }^{[4]}\end{array}$ \\
\hline high levels of total LDH, and/or isoforms ( 3,4 and/or 5) & 14 & 6 & 2 \\
\hline High levels of Ca125 & 12 & 5 & 3 \\
\hline Central vasculature at ECD examination & 14 & 5 & 0 \\
\hline
\end{tabular}

\subsection{I nclusion criteria}

Patients suffering from uterine "mass" waiting for surgery, that are considered at risk and/or of uncertain clinical laboratory investigations and imaging interpretation (US).

The risk is defined by:

- onset of uterine masses in postmenopausal women;

- $\quad$ rapid increase in size (> 6 weeks in 9-12 months) ${ }^{[30]}$;

- failure of feedback to the treatment with GnRH analogues;

- $\quad$ high levels of total LDH, and / or isoforms (3, 4 and/or 5);

- $\quad$ high levels of Ca125;

- $\quad$ presence of central and peripheral vasculature, with a low flow resistance, high systolic peak at echo-color Doppler examination (ECD) ${ }^{[31]}$.

All patients expressed informed consent.

\subsection{Exclusion criteria}

Uncooperative patients or with contraindications to MRI examination.

The same patients have later undergone surgery laparotomic or laparoscopic myomectomy or hysterectomy, so that the clinical, biochemical, and ultrasound data and the one of MRI preoperative could have been related to the final histology data.

Among the 42 patients: 
- 14 have undergone laparoscopic myomectomy with protected morcellation;

- 4 have been subjected to laparoscopic hysterectomy;

- 8 have undergone staging surgery (laparotomy with peritoneal washing + bilateral hysteroannessiectomy + lymphadenectomy);

- 2 have been subjected to hysteroscopic biopsy;

- 2 have been subjected to hysteroscopic biopsy + resectoscopy with metroplasty;

- 12 have been subjected to laparoscopic hysterectomy.

These patients had more uterine lesions but we evaluated the most suspicious lesions.

\subsection{MRI protocol}

Before the MRI investigation, the patients were request to fast for at least 4-6 hours. Few minutes before the test, an antiperistaltic was administered (i.m.) in order to limit motion artefact of the small intestine and a moderate bladder distension. Patients were placed supine. The sequences were acquired through a magnet with high field strength (Philips InteraAchieva $1.5 \mathrm{~T}$ ) and phased-array coils reaching an excellent anatomic detail of the structures under consideration.

The study protocol includes a morphological study by the acquisition of turbo spin-echo sequences (TSE) T1-weighted, T2-weighted, IR sequences and DWI using three factors of b-values $\left(0,400,800 \mathrm{~s} / \mathrm{mm}^{2}\right)$ then it is undertaken a dynamic study with 3D T1 FAT SAT sequences before and after the administration of paramagnetic contrast agent, Gd-BOPTA administered at a dose of $0.1 \mathrm{ml} / \mathrm{kg}$ via an automatic injector (Medrad) with a flow of $2 \mathrm{ml} / \mathrm{sec}$, according to the sizes of the axial, sagittal and coronal space. The dynamic study consists of six stages: one using a pre-contrast medium and the others five post-contrastographics.

The sequences acquired at 20,60,120,150 and 180 seconds after the administration of a.d.c. represent another criterion in the differential diagnosis between sarcomas and uterine leiomyomas, because it has been demonstrated that sarcomas

show an intense and avid enhancement with respect to the benign formations ${ }^{[27-29]}$. In addition, in the post-processing it was possible to process the captured images through subtraction and calculation of time-intensity curves. The regions of interest (ROI) were positioned in the areas of identified enhancement, and we performed a "semi-quantitative" analysis, which consists of a numerical measurement of the signal intensity over a period of time, thus creating the time-intensity curves.

\subsection{I mage analysis}

Through the morphological imaging, we've evaluated: the seat, the maximum size on three orthogonal planes, the contours (rounded, oval or lobulated), margins (regular or irregular), the components within the lesion (hemorrhagic, cystic, cellular, hyaline, mixoid areas); the presence of fluid effusion, enlarged lymph nodes, the relationship with the surrounding structures. We've also evaluated the signal growth to high values of b-value on DWI sequences. Finally, we've evaluated the contrastographic behaviour of lesions. In particular, after the injection of a.d.c., the level of enhancement of the lesion has been defined as follows:

- 1 mild enhancement (less than the myometrium); 2 moderate enhancement (as the myometrium); 3 intense enhancement (greater than the myometrium);

- $\quad$ Also time-signal intensity curves have been taken into account;

- One radiologist, blinded to the histological results independently, analyzed MRI datasets of each participant. 


\section{Results}

Of the 42 patients who underwent surgery, the histological examination gave the following results:

- 14 degenerated leiomyomas;

- 8 sarcomas (4 carcinosarcomas and 4 stromal sarcomas polymorphs of which one of high-grade);

- 16 cellular leiomyomas;

- 2 malformation of mullerian ducts (uterine septum) with associated adenomyosis;

- 2 adenomyosis.

\section{MRI features}

The different MRI features, DWI and enhancement pattern are shown in Table 3.

Table 3. Comparison of different MRI features, DWI and enhancement pattern

\begin{tabular}{lll}
\hline Variable & Leiomyomas (30 cases) & Sarcomas $^{[8]}$ \\
\hline Tumor margins & 30 & 0 \\
regular & 0 & 8 \\
irregular & $80 \mathrm{~mm}$ & $100 \mathrm{~mm}$ \\
Size (mm) & & 0 \\
T2-weighted signal & 4 & 8 \\
Low & 26 & 8 \\
Intermediate & 26 & 0 \\
T2-weighted signal heterogeneity & 1 & 8 \\
Intra-tumoral haemorrhage & 16 & 8 \\
High b 800 signal & & 0 \\
Heterogeneous enhancement Dynamic contrast enhanced & 4 & \\
early & 26 & \\
later & & \\
\hline
\end{tabular}

Four of the eight sarcomas were carcinosarcoma, all of voluminous dimensions of which one at the stage IV, all of voluminous dimensions. The largest new formation measured $12.6 \mathrm{~cm} \times 3.8 \mathrm{~cm} \times 5.2 \mathrm{~cm}(\mathrm{D} . \mathrm{L} \times$ D.AP $\times$ DT); it had irregular borders, extended from the uterine fundus to the cervix with the commitment of the endometrial cavity and showed heterogeneous signal intensity due to the presence of fluid components hyperintense on T2 images and solid components hypointense on T1 and T2 images (see Figure 1). It also marked and displaced the bladder formerly-cranially and contracted relations of contiguity/continuity whit the rectosigmoid. Finally in the left iliac and bilateral inguinal regions, there were enlarged lymph nodes and discreet payment extended in the abdominal and pelvic regions. The other sarcomas had the edge not always well-defined, showed etherogeneous hypointense signal on T1 a hyperintense area on T2.

Of the four endometrial stromal sarcomas polymorphic, one was at stage IV. In this case the uterus was no longer identifiable and there was a voluminous formation of $11 \mathrm{~cm} \times 12 \mathrm{~cm} \times 12 \mathrm{~cm}($ D.L $\times$ D.A.P $\times$ D.T) with irregular margins and heterogeneous structure for the presence of solid components, that were hypointense on T1 and T2, frankly liquid components, hypointense on $\mathrm{T} 1$ and hyperintense on T2 and serum/protein as hyperintense on T1. This formation displaced formerly the bladder and bowel loop and then contracted relations of contiguity/continuity with the rectosigmoid. Finally in the bilateral inguinal enlarged there were lymph nodes and a thin fluid collection in the left iliac fossa. The other 
formations showed inhomogeneous hypointense signal on T1 images with hyperintense areas on T2/IR and impressed the endometrial cavity.
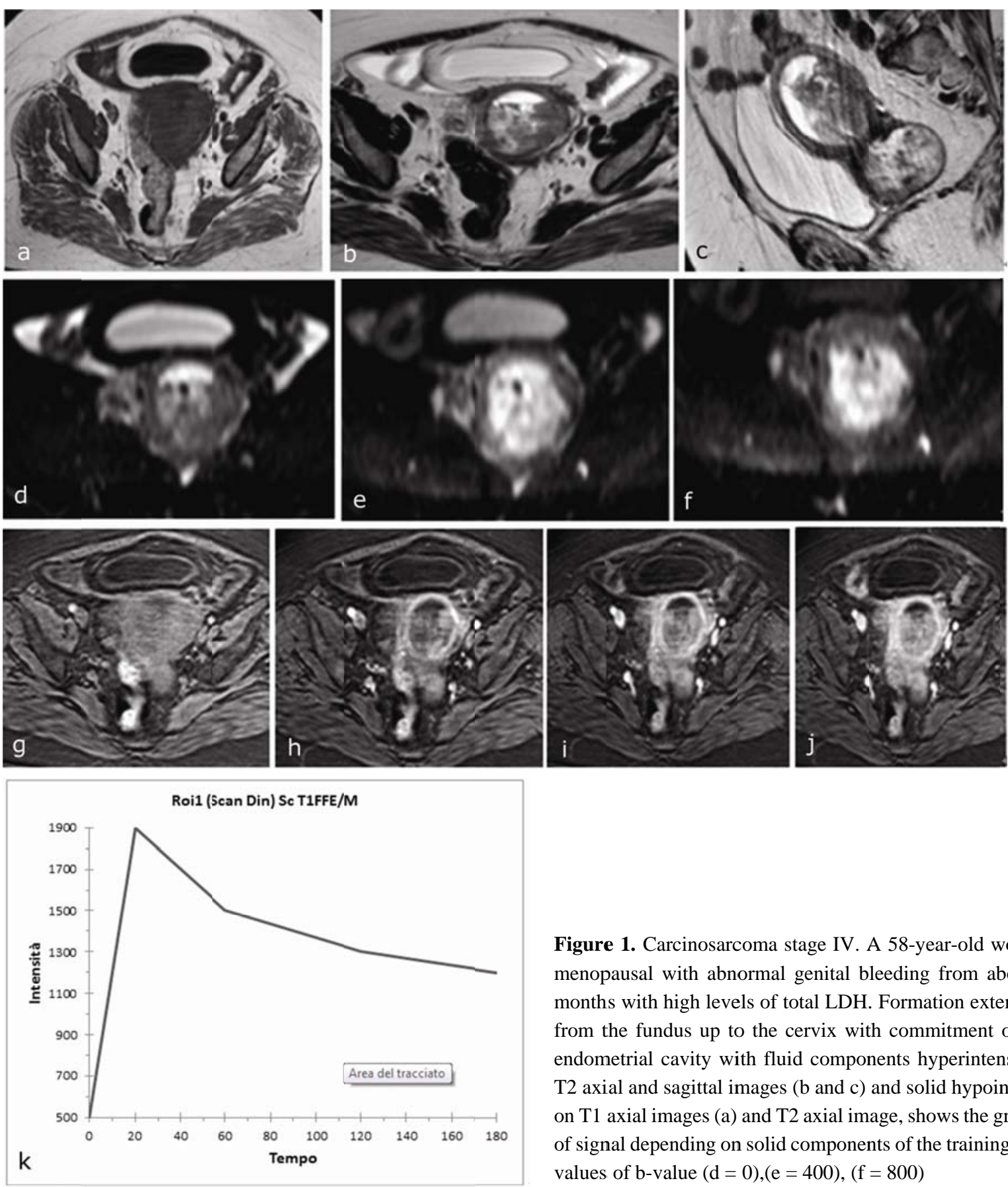

Figure 1. Carcinosarcoma stage IV. A 58-year-old woman menopausal with abnormal genital bleeding from about 3 months with high levels of total LDH. Formation extending from the fundus up to the cervix with commitment of the endometrial cavity with fluid components hyperintense on T2 axial and sagittal images (b and c) and solid hypointense on T1 axial images (a) and T2 axial image, shows the growth of signal depending on solid components of the training high values of b-value $(d=0),(e=400),(f=800)$

Of the fourteen degenerated leiomyomas all presented themselves as massive formations $(7 \mathrm{~cm}-11 \mathrm{~cm})$ rounded, uneven, 13 of which appeared hypointense on T1 images and T2 images with striae and hyperintense areas on T2/IR images (see Figure 2), while only 1 case had no streaks and spots hyperintense on T1 images as content from serum/protein hemorrhagic. In 10 degenerated leiomyomas, margins appeared well defined, while in 4 cases they came out to be hazy. Enlarged lymph nodes were not present in any case, and in four cases there was a flap of payment in the pelvic. 

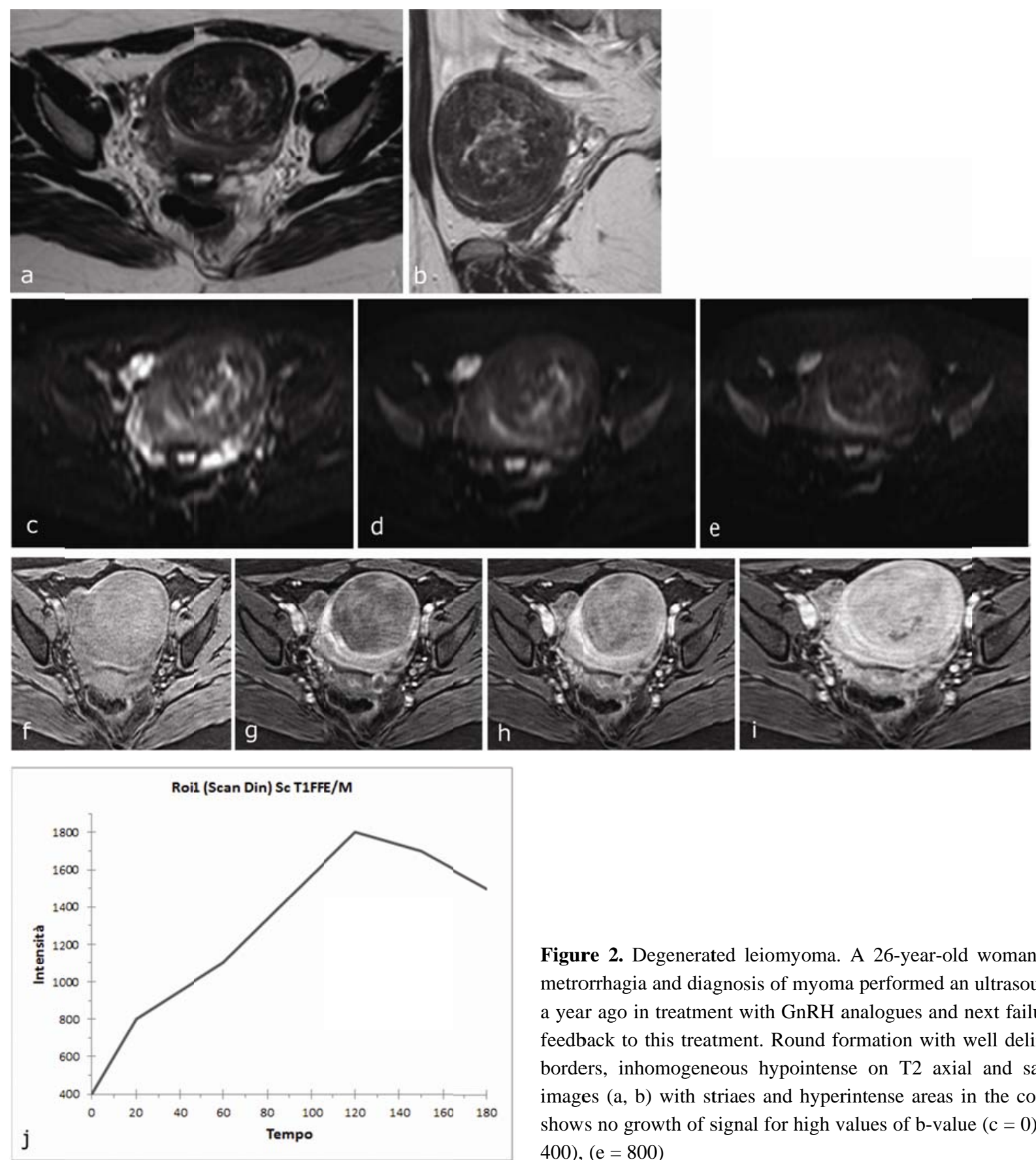

Figure 2. Degenerated leiomyoma. A 26-year-old woman with metrorrhagia and diagnosis of myoma performed an ultrasound to a year ago in treatment with GnRH analogues and next failure of feedback to this treatment. Round formation with well delimited borders, inhomogeneous hypointense on T2 axial and sagittal images (a, b) with striaes and hyperintense areas in the context, shows no growth of signal for high values of b-value $(c=0),(d=$ 400), $(\mathrm{e}=800)$

Of the sixteen cellular leiomyomas all the formations showed smooth margins; 15 had bulky size $(5 \mathrm{~cm}-7 \mathrm{~cm})$, while only one was centimeter (see Figures 3, 4). Twelve appeared hypointense on T1 images and T2 images with striaehyperintense on T2/IR images, while 4 were isointense to myometrium on T1 images and hypointense on T2/IR images. In no one case enlarged lymph nodes were present and in 4 cases there was a flap of payment in the pelvic.

Of the 4 patients of where MRI did not show the presence of neo-formations: two presented an anomaly of Muller's duct and a widely uneven appearance of the myometrium with thickening of the junctional zone of about $12 \mathrm{~mm}$ associated with cystic areolas, signal of adenomyosis; two cases had a negative outcome of the MRI exam was negative (uterus in AVF within the dimensional limits of the standard and good differentiation of the zonal anatomy without alterations of 
focus in the context) and instead we performed biopsies draw blood during hysteroscopy and the outcome of histological analysis showed adenomyosis.
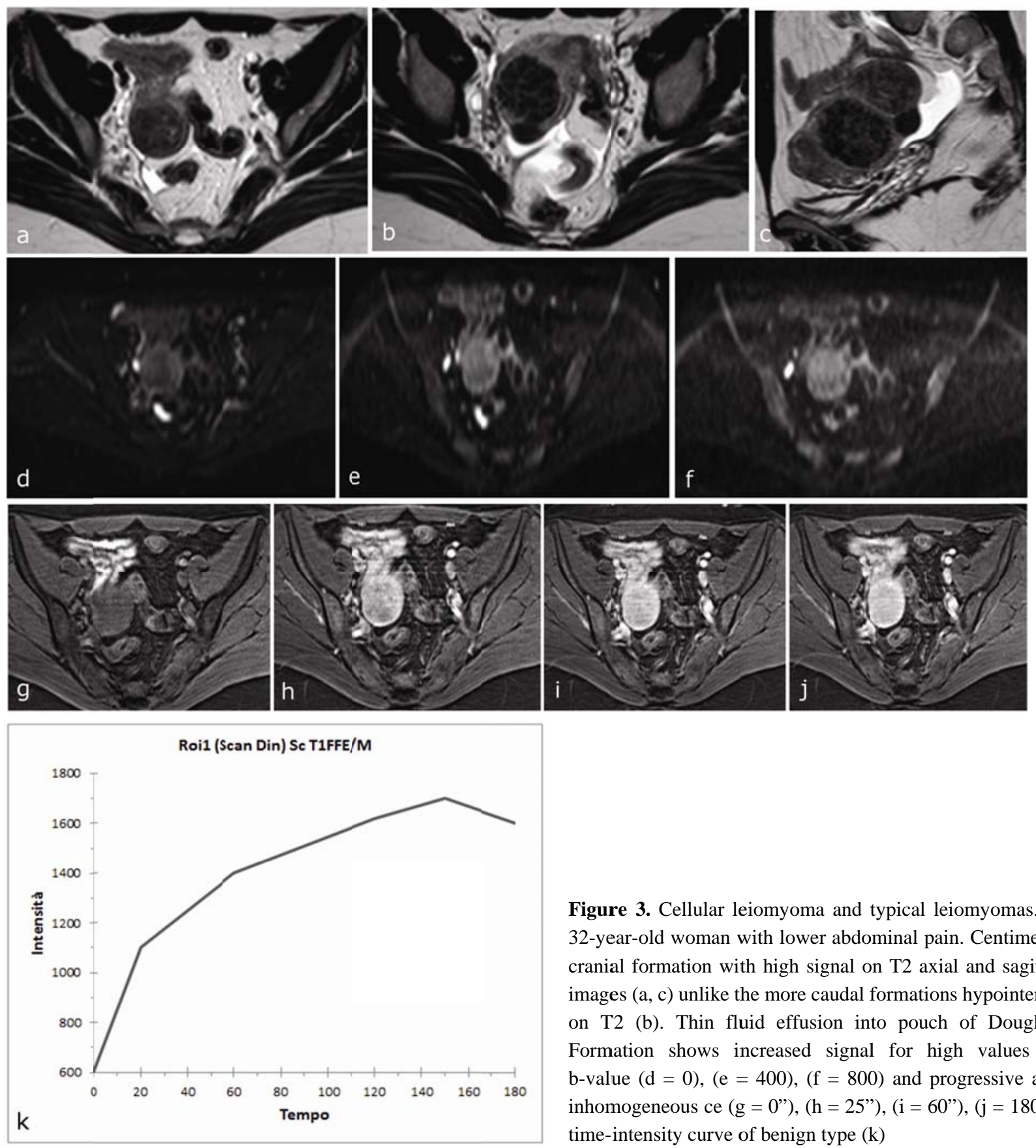

Figure 3. Cellular leiomyoma and typical leiomyomas. A 32-year-old woman with lower abdominal pain. Centimeter cranial formation with high signal on T2 axial and sagittal images (a, c) unlike the more caudal formations hypointense on T2 (b). Thin fluid effusion into pouch of Douglas. Formation shows increased signal for high values of b-value $(\mathrm{d}=0),(\mathrm{e}=400),(\mathrm{f}=800)$ and progressive and inhomogeneous ce ( $\mathrm{g}=0$ ”), $(\mathrm{h}=25$ ”), $(\mathrm{i}=60$ ”), $(\mathrm{j}=180$ ”), time-intensity curve of benign type $(\mathrm{k})$

The eight sarcomas showed hypersignal for high values of b-value in particular at the level of solid components (see Figure 1).

The 14 degenerated leiomyomas showed no hypersignal for high values of b-value (see Figure 2).

The sixteen cellular leiomyomas showed hypersignal for high values of b-value (see Figures 3, 4). 

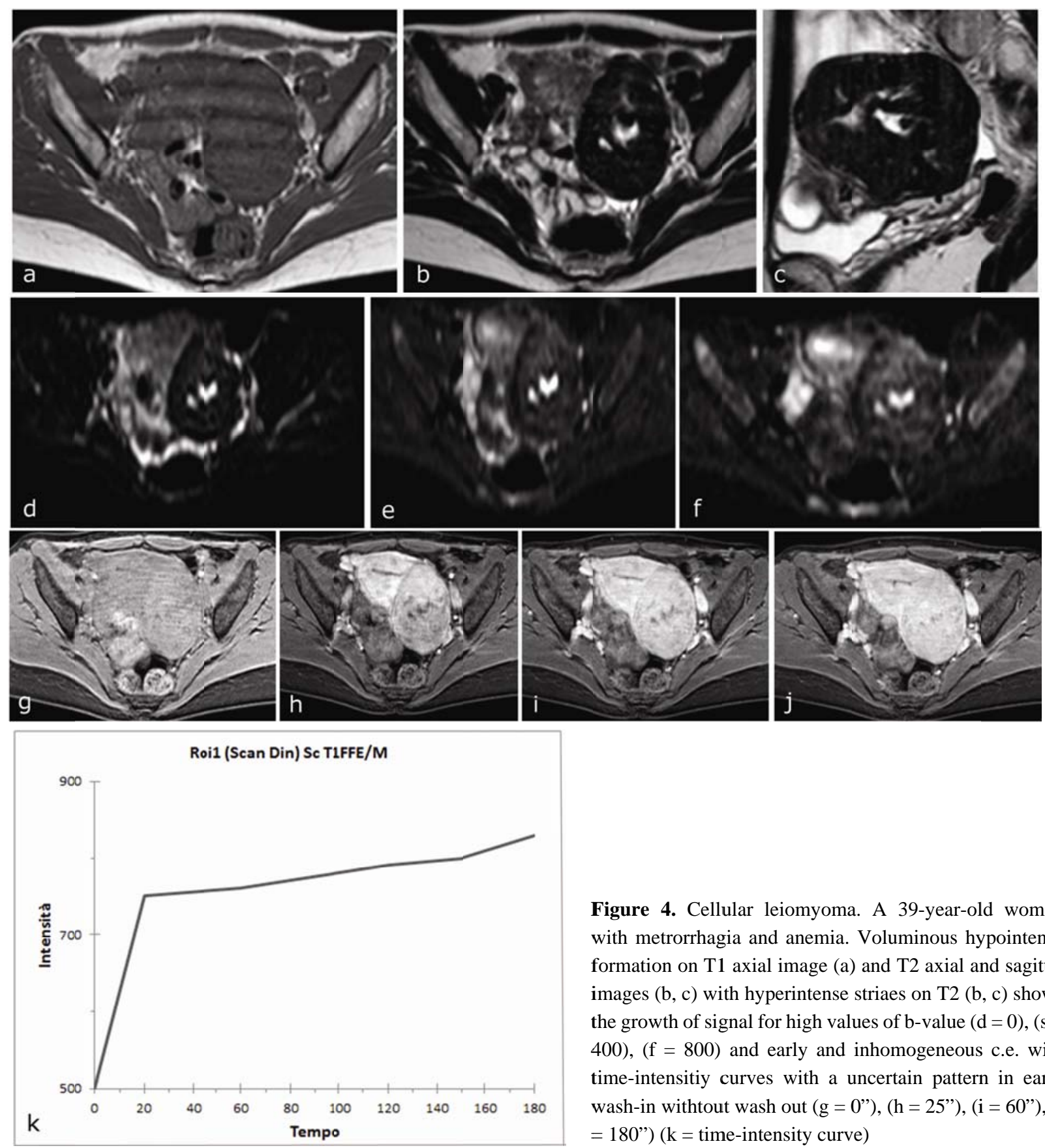

Figure 4. Cellular leiomyoma. A 39-year-old woman with metrorrhagia and anemia. Voluminous hypointense formation on T1 axial image (a) and T2 axial and sagittal images (b, c) with hyperintense striaes on T2 (b, c) shows the growth of signal for high values of $b$-value $(d=0),(s=$ 400), ( $f=800$ ) and early and inhomogeneous c.e. with time-intensitiy curves with a uncertain pattern in early wash-in withtout wash out ( $\mathrm{g}=0$ ”), $(\mathrm{h}=25$ ”), (i = 60”), (j $=180$ ”) $(\mathrm{k}=$ time-intensity curve $)$

The eight sarcomas showed an early and intense c.e after i.v. infusion of a.d.c. (see Figure 1) with time-intensity curves with pattern of malignant type characterized by rapid wash-in and wash-out fast.

The 14 degenerated leiomyomas showed progressive tenuous and uneven c.e. after i.v. infusion of a.d.c. (see Figure 2), with time-intensity curves with pattern of benign type characterized by slow and progressive wash-in without a clear washout .

Of the sixteen cellular leiomyomas:

- 8 showed progressive and inhomogeneous c.e. (see Figure 3); 
- $\quad 2$ showed nuanced and progressive c.e.;

- 2 showed progressive and more evident in the late stages c.e.;

with time-intensity curves with pattern of benign type characterized by slow and progressive wash-in without a clear washout.

Only for four cellular leiomyomas whose morphology was correctly directed by-MRI, the post-contrast-MRI offered different possible interpretation: the formations, indeed, showed early and inhomogenous c.e. with time-intensity curves with type doubt pattern characterized by early wash-in without obvious wash-out (see Figure 4).

\section{Discussion}

Leiomyomas are the most common benign uterine tumors in young women. They occur in $20 \%$ of women over 30 years old.

Uterine sarcomas are rare tumors representing approximately $1 \%$ of cancers of the female genitalia and 3\%-7\% of all uterine cancers ${ }^{[5]}$. To date there is no sufficient and sensitive method to allow an accurate preoperative differential diagnosis between myoma and sarcoma yet.

However, recent studies suggest that MRI through diffusion and post-contrastographic sequences, represent emerging valuable technique for the differential diagnosis between degenerate leiomyoma and sarcomas ${ }^{[21,22,25-28]}$.

According to some authors, high signal intensity in DWI in malignant tissue is attributed to his topathological characteristics, including hypercellularity, enlargement of nuclei, hyperchromatisms and angulation of the nuclear contour, that result in a reduction of diffusional displacement of water molecules ${ }^{[23]}$.

Leiomyomas showed variable signal intensity on T2-weighted images, and may reflect a wide variety of histological features depending on degeneration or cellular content with abundant hyalinised collagen.

Tamai et al. ${ }^{[22,23]}$ demonstrated that cellular leiomyomas may not be distinguished from sarcomas based on DWI by increased cellularity. On the other hand, degenerated leiomyomas tended to exhibit low SI on DWI compared with sarcomas.These results mayreflect the presence of abundant water content within thelesions. A ccordingly, DWI can aid inthe differentiation between degenerated leiomyomas andsarcomas.

According to some authors, in our study the cellular leiomyomas and sarcomas showed hypersignal for high values of b-value. Instead, the degenerated leiomyomas showed no hypersignal for high values of b-value.

According to some authors, even post-contrastographic sequences allow to obtaine more precise data compared to DWI sequences ${ }^{[29]}$.

Lin et al. demonstrated that CE-MRI yielded significantly higher diagnostic accuracy and specificity as compared with DWI, in differentiation between sarcomas and benign leiomyomas ${ }^{[29]}$.

The sarcomas showed early and avid enhancement on contrast-enhanced images ${ }^{[24]}$.

In our study, the sarcomas showed an early and intense enhancement on contrast-enhanced images with time-intensity curves with pattern of malignant type characterized by rapid wash-in and wash-out fast. The 26 leiomyomas showed progressive tenuous and uneven enhancement on contrast-enhanced images, with time-intensity curves with pattern of benign type characterized by slow and progressive wash-in without a clear washout. 
Only four cellular leiomyomas showed early and in homogenous enhancement on contrast-enhanced images with time-intensity curves with type doubt pattern characterized by early wash-in without obvious wash-out.

The true positive, false positive, true negative and false negative results are shown in Table 4.

Table 4. True positive (TP), false positive (FP), true negative (TN) and false negative (FN) results

\begin{tabular}{|l|l|l|}
\hline & $\mathrm{M}^{+}$ & $\mathrm{M}-$ \\
\hline $\mathrm{T}^{+}$ & $8(\mathrm{TP})$ & $4(\mathrm{FP})$ \\
\hline $\mathrm{T}-$ & $0(\mathrm{FN})$ & $30(\mathrm{TN})$ \\
\hline
\end{tabular}

Statistical analysis of our study shows an increased sensitivity of $100 \%$ in using diffusion-weighted and postcontrastographic sequences compared to morphological examination, a specificity of about $88 \%$, a PPV of about $66 \%$, a NPV of about $100 \%$ and a diagnostic accuracy of about $90 \%$.

We cathegorized leiomyomas into histopathological subtypes. Our population study include patients with other benign myometrial conditions, such as adenomyosis.

Our results show that combined DWI and post-contrastographic sequences is better than DWI alone in the differentiation of uterine sarcomas from benign leiomyomas, especially from cellular leiomyomas.

However the limitations of this study are represented by the low number of reported uterine sarcomas and by the size of the sample. Thus, a larger study should be prospectively performed to verify our results.

\section{Conclusions}

In clinically uncertain cases, DWI and dynamic sequences allowed us to distinguish sarcomas from cellular and degenerate leiomyomas as sarcomas showed hypersignal in diffusion-weighted sequences and early and high c.e. in post-contrastographic sequences, with time-intensity curves of malignant type.

The DWI along with dynamic sequences could be a valuable tool to support radiologists in the differential diagnosis of atypical leiomyoma, cellular and uterine sarcomas. Such data may allow the gynecologists the most appropriate approach to a single patient.

\section{Conflicts of interest disclosure}

The authors have declared no conflicts of interest.

\section{References}

[1] Ueda H, Togashi K, Konishi I, et al. Unusual Appearances of Uterine Leiomyomas: MR Imaging Findings and Their Histopathologic Backgrounds. Radio Graphics. 1999; 19: S131-S145. PMid:10517450

http://dx.doi.org/10.1148/radiographics.19.suppl_1.g99oc04s131

[2] Murase E, Siegelman ES, Outwater EK, et al. Uterine leiomyomas: Histopathologic Features, MR Imaging Findings, Differential Diagnosis, and treatment. Radio Graphics. 1999; 19: 1179-1197. PMid:10489175

http://dx.doi.org/10.1148/radiographics.19.5.g99se131179

[3] Orazio T, Nicoletta C, Stefano M, et al. Role of MRI for Evaluation of Uterine Giant Myoma: Case Report. International Journal of Medical Imaging. 2015; 3(3): 59-62. http://dx.doi.org/10.11648/j.ijmi.20150303.13

[4] Deshmukh SP, Gonsalves CF, Guglielmo FF, et al. Role of MR Imaging of Uterine leiomyomas before and after Embolization. Radio Graphics. 2012; 32: E 251-281.

[5] D’Angelo E, Prat J. Uterine Sarcomas: A Review. Gynecologic Oncology. 2010; 116(1): 131-9. 
[6] Sutton G. Uterine Sarcomas 2013. Gynecologic Oncology. 2013; 130(1): 3-5.

[7] Park JY, Park SK, Kim DY, et al. The impact of tumor morcellation during surgery on the prognosis of patients with apparently early uterine leiomyosarcoma. Gynecologic Oncology. 2011; 122(2): 255-259.

[8] Bogani G, Cliby WA, Aletti GD, et al. Impact of morcellation on survival outcomes of patients with unexpected uterine leiomyosarcoma: A systematic review and meta-analysis. Gynecologic Oncology. 2015; 137(1): 167-172.

[9] FDA. Laparoscopic Uterine Power Morcellation in Hysterectomy and Myomectomy: FDA Safety Communication. 2014; Available from http://www.fda.gov/medicaldevices/safety/alertsandnotices/ucm393576.htm

[10] Brölmann H, Tanos V, Grimbizis G, et al. Option on fibroid morcellation: a literature review. Gynecol Surg. 2015 ; $12(1)$ : 3-15. PMid:25774118 http://dx.doi.org/10.1007/s10397-015-0878-4

[11] Ulrich LG. Benign leiomyoma or malignant sarcoma: The difficult differential diagnosis. Maturitas. 2015.

[12] Bonneau C. Value of ultrasonography and magnetic resonance imaging for the characterization of uterine mesenchymal tumors. Acta Obstetricia et Gynecologica Scandinavica. 2014; 93(3): 261-268.

[13] Foti PV. Imaging RM dei leiomiomi uterini. Il giornale italiano di Radiologia Medica. 2015; 2: 35-48.

[14] Aviram R, Ochshorn Y, Markovitch O, et al. Uterine Sarcoma Versus Leiomyomas: Gray-scale and Doppler Sonographic Findings. Journal of Clinical Ultrasound. 2005; 33(1): 10-3.

[15] Exacoustos C, Romanini ME, Amadio A, et al. Can Gray-Scale and Color Doppler Sonography Differentiate between Uterine Leiomyosarcoma and Leiomyoma? Journal of Clinical Ultrasound. 2007; 35(8): 449-57.

[16] Stewart EA. Differentiating uterine leiomyomas (fibrods) from uterine sarcomas. Up to Date Dicembre. 2013.

[17] Amant F, Coosemans A, Debiec-Rychter M, et al. Clinical management of uterine sarcomas. Lancet Oncol. 2009 ; $10: 1188$. http://dx.doi.org/10.1016/S1470-2045(09)70226-8

[18] Dueholm M, Lundorf E, Hansen ES, et al. Accuracy of magnetic resonance imaging and transvaginal ultrasonography in the diagnosis, mapping, and measurement of uterine myomas. Am J Obstet Gynecol. 2002; 186(3): 409-15. PMid:11904599 http://dx.doi.org/10.1067/mob.2002.121725

[19] Cornfeld D, Israel G, Martel M. MRI appearance of mesenchimal tumours of the uterus. European Journal of Radiology. 2010; 74(1): 241-249.

[20] Tirumani SH, Ojili V, Shanbhogue AKP, et al. Current concepts in the imaging of uterine sarcoma. Abdom Imaging. 2013; 38(2): 397-411. http://dx.doi.org/10.1007/s00261-012-9919-x

[21] Thomassin-Naggara I, Dechoux S, Dechoux B, et al. How to differentiate benign from malignant myometrial tumours using MR Imaging. Eur Radiol. 2013: 23(8): 2306-2314. http://dx.doi.org/10.1007/s00330-013-2819-9

[22] Tamai K, Koyama T, Saga T, et al. The utility of diffusion-weighted MR imaging for differentiating uterine sarcomas from benign leiomyomas. Eur Radiol. 2008; 18(4): 723-730. PMid:17929022 http://dx.doi.org/10.1007/s00330-007-0787-7

[23] Namimoto T, Yamashita Y, Awai K, et al. Combined use of T2-weighted and diffusion-weighted 3-T MR imaging for differentiating uterine sarcomas from benign leiomyomas. Eur Radiol. 2009; 19: 2756-2764. PMid:19504102 http://dx.doi.org/10.1007/s00330-009-1471-x

[24] Zhang H, Zhang GF, He ZY, et al. Evaluation of primary adnexal masses by 3T MRI: categorization with conventional MR imaging and diffusion-weighted imaging. Journal of Ovarian Research. 2012; 5: 33. http://dx.doi.org/10.1186/1757-2215-5-33

[25] Sato K, Yuasa N, Fujita M, et al. Clinical application of diffusion-weighted imaging for preoperative differentiation between uterine leiomyoma and leiomyosarcoma. Am J Obstet Gynecol. 2014; 210(4): 368.e1-8. http://dx.doi.org/10.1016/j.ajog.2013.12.028

[26] Dhanda S, Thakur M, Kerkar R, et al. Diffusion-weighted Imaging of GynecologicTumors: Diagnostic Pearls and Potential Pitfalls. Radio Graphics. 2014; 34(5): 1393-1416. http://dx.doi.org/10.1148/rg.345130131

[27] Zhang GF, Zhang H, Tian XM, et al. Magnetic resonance and diffusion-weighted imaging categorization of uterine sarcomas: correlation with pathological findings. Clinical Imaging. 2014; 38(6): 836-844.

[28] Goto A, Takeuchi S, Takeuchi K, et al. Usefulness of Gd-DTPA contrast-enhanced dynamic MRI and serum determination of $\mathrm{LDH}$ and its isozymes in the differential diagnosis of leiomyosarcoma from degenerated leiomyoma of the uterus. Int $\mathrm{J}$ Gynecol Cancer. 2002; 12(4): 354-361. PMid:12144683 http://dx.doi.org/10.1046/j.1525-1438.2002.01086.x

[29] Lin G, Yang LY, Huang YT, et al. Comparison of the Diagnostic Accuracy of Contrast-Enhanced MRI and Diffusion-Weighted MRI in the Differentiation Between Uterine Leiomyosarcoma/Smooth Muscle Tumor With Uncertain Malignant Potential and Benign Leiomyoma. J Magn Reson Imaging. 2015; 47(3): 249-278.

[30] Wallach EE, Buttram Jr VC, Reiter RV, et al. Uterine leiomyomata: etiology, symptomatology, and management. Fertil Steril. 1981; 36(4): 433-45. http://dx.doi.org/10.1016/S0015-0282(16)45789-4

[31] Exacoustos C, Romanini ME, Amadio A, et al. Can Gray-Scale and Color-Doppler Sonography Differentiate between Uterine Leiomyosarcoma and Leiomyoma? Journal of Clinical Ultrasound. 2007; 35(8): 449-57. 